\title{
Inter-quark potentials from NBS amplitudes and their applications
}

\author{
Hideaki lida* \\ Department of Physics, Kyoto University, Sakyo-ku, Kyoto 606-8502, Japan \\ Mathematical Physics Laboratory, RIKEN Nishina Center, Wako, Saitama 351-0198, Japan \\ E-mail: hiida@riken.jp
}

\section{Yoichi lkeda}

Department of Physics, Tokyo Institute of Technology, Meguro-ku, Tokyo 152-8551, Japan

E-mail: yikeda@riken.jp

\begin{abstract}
Inter-quark potentials at finite temperature are derived from Nambu-Bethe-Salpeter (NBS) amplitudes. Employing the formalism developed by Laine et al. and Beraudo et al., we calculate the $\bar{Q} Q$ potentials with finite quark masses in imaginary-time. The extracted potentials show Coulomb plus linear behavior, and have moderate temperature dependence below $T_{c}$. On the other hand, the potential considerably changes and becomes flat at long distance above $T_{c}$. Although our potential is obtained in imaginary-time, it is expected that the obtained potential is a natural extension of potentials from the Polyakov loop correlations.
\end{abstract}

The XXIX International Symposium on Lattice Field Theory - Lattice 2011

July 10-16, 2011

Squaw Valley, Lake Tahoe, California

${ }^{*}$ Speaker. 


\section{Introduction}

Inter-quark potentials have physical implications of color confinement and hadron spectroscopy. Regge slope indicates that the inter-quark potentials have the linear part with string tension $\sigma \simeq$ $1.3 \mathrm{GeV}$ at long distance. To understand the origin of the linear part is an important issue of the hadron physics. On the other hand, at short distance, the inter-quark potentials are expected to have the Coulomb interaction, which is suggested by the analogy between quarkonium and positronium. Actually, the Coulomb plus linear potential reproduces the low-lying hadron spectra well in quark models.

In Ref. [1], we propose a new method to extract the inter-quark potentials with finite quark masses from lattice QCD. We utilize the Nambu-Bethe-Salpeter (NBS) wave functions of quarkanti-quark systems for this purpose, which has been recently developed by HAL QCD Collaboration to extract nuclear force on lattice [2]. The obtained inter-quark potentials show the Coulomb plus linear behavior, and the string tension for heavy quark case is consistent with one derived from Wilson loop. In Ref. [3], the authors perform detailed analyses of the inter-quark potential of charmonium in $2+1$ flavors at almost physical quark mass. They find the obtained potential is very close to the inter-quark potential in non-relativistic potential model in Ref.[4]. From these evidences, the method seems to work reasonably well.

At finite temperature, inter-quark potentials give important information for the dissociation of heavy quarkonium. A famous signature of Quark-Gluon-Plasma (QGP), $J / \psi$ suppression, is closely related to the potential, for example. There are several studies of direct measurement of heavy quarkonia on the lattice at finite temperature $[5,6,7,8]$. However, in spite of much effort, there is still no common view for the dissociation temperature of these quarkonia. In Ref. [5, 6, 7], they calculate temporal correlators of quarkonia and reproduce spectral functions by using Maximal Entropy Method (MEM). The dissociation temperature of $J / \psi$ and $\eta_{c}$ is $T=(1.6-1.8) T_{c}$, where $T_{c}$ is the critical temperature of QCD, in Ref. [6]. In Ref. [7], the dissociation of $J / \psi$ and $\eta_{c}$ occurs very moderately, and even above $2 T_{c}$, there are still small peaks of these states. On the other hand, there is another method of studying quarkonia above $T_{c}$, in which effective masses derived from the qurakonium correlator are used [8,9]. In this method, dissociation of $J / \psi$ and $\eta_{c}$ does not occur even around $2 T_{c}$. Furthermore, a study from QCD sum rule using MEM [10] shows very early dissociation of $J / \psi$. Under the circumstance, information of the inter-quark potential in heavy quarkonium at finite temperature would help us to understand the dissociation problem.

In this paper, we study the inter-quark potential of heavy quarkonia at finite temperatures from those NBS amplitudes. We extend the method obtaining inter-quark potentials from NBS amplitudes at zero temperature to finite temperature systems. Our formalism is similar to that proposed by Laine et al. [11] and Beraudo et al. [12], although ours is formulated with finite quark masses.

The paper is organized as follows. In Sec. 2, the studies of Laine et al. and Beraudo et al. are briefly reviewed and then our formalism is explained. In Sec. 3, we show the simulation setup and numerical results of the potentials. Section 4 is devoted to discussion and summary of the paper. 


\section{Formalism of extraction of inter-quark potentials with finite masses at finite temperature}

In this section, we briefly review the formalism to extract the inter-quark potentials of the vector channel with finite quark masses $(J / \psi)$ at finite temperature, which was developed in Ref. [11] and [12]. According to Ref. [11], the inter-quark potential at finite temperature is defined by starting with the following quark-anti-quark correlation function:

$$
\check{C}_{>}(t, \vec{r}) \equiv \sum_{\vec{x}}\left\langle\bar{q}(t, \vec{x}+\vec{r}) \gamma^{\mu} W(t, \vec{x}+\vec{r} ; t, \vec{x}) q(t, \vec{x}) \bar{q}(0, \overrightarrow{0}) \gamma_{\mu} q(0, \overrightarrow{0})\right\rangle
$$

where $W_{\vec{r}}\left[t, \vec{x}_{1} ; t_{0}, \vec{x}_{0}\right]$ is the Wilson line, which connects $\left(t_{0}, \vec{x}_{0}\right)$ to $\left(t_{1}, \vec{x}_{1}\right)$ along a straight path in space-time. In the case of non-interacting heavy quark-anti-quark pair, the quark-anti-quark correlation function in Eq. (2.1) satisfies the Schrödinger-type equation:

$$
\left[i \partial_{t}-\left(2 M-\frac{\nabla_{\vec{r}}^{2}}{M}+\mathscr{O}\left(\frac{1}{M^{3}}\right)\right)\right] \check{C}_{>}(t, \vec{r})=0
$$

With the presence of the interactions between quarks, one can expect that the quark-anti-quark correlation function in Eq. (2.1) satisfies the Schrödinger-type equation with the proper temperaturedependent inter-quark potential at finite temperature $V(t, \vec{r} ; T)$ :

$$
\left[i \partial_{t}-\left(2 M-\frac{\nabla_{\vec{r}}^{2}}{M}+V(t, \vec{r} ; T)+\mathscr{O}\left(\frac{1}{M^{3}}\right)\right)\right] \check{C}_{>}(t, \vec{r})=0 .
$$

Using Eq. (2.3), the proper potentials $V(t, \vec{r} ; T)$ are inversely extracted through the quark-anti-quark correlation function $C_{>}(t, \vec{r})$.

In Ref. [11], the static potential, which is the proper potential with infinitely heavy quarks, is calculated through the Wilson loop in Euclidean space-time:

$$
C_{E}(\tau, \vec{r}) \equiv \frac{1}{N_{c}} \operatorname{Tr}\langle W(0, \vec{r} ; \tau, \vec{r}) W(\tau, \vec{r} ; \tau, \overrightarrow{0}) W(\tau, \overrightarrow{0} ; 0, \overrightarrow{0}) W(0, \overrightarrow{0} ; 0, \vec{r})\rangle
$$

within the Hard-Thermal Loop approximation. After the analytic continuation into Minkowski space-time, one finds the static potential $V_{>}(t, \vec{r} ; T)$ through the Schrödinger-type equation, $i \partial_{t} C_{>}(t, r)=$ $V_{>}(t, r ; T) C_{>}(t, r)$. Then, the static potential at the large-time $\operatorname{limit} \lim _{t \rightarrow \infty} V_{>}(t, r ; T)$, gives not only a real part but also an imaginary part. The real part corresponds to Debye screening and the imaginary part to Landau damping of low-frequency gauge fields that mediate interactions between the two heavy quarks. Thus, the definition of the potential at finite temperatures seems to be adequate.

Also, in Ref. [12], the heavy quark potential in hot QED plasma is investigated in the Coulomb gauge. The calculations of the potential in real-time direction at $t \rightarrow \infty$ and imaginary-time direction at $\tau=\beta$ reveals that the real part of these potentials coincides, which correspond to Debye screening. On the other hand, the imaginary part of the potential appears only in the real-time case.

In this study, the formalism in Refs. [11] and [12] is extended to the case of the inter-quark potential with finite quark masses in the vector channel, where all the effects of the finite quark 


\begin{tabular}{cccccc}
\hline \hline lattice size & $\beta$ & $a_{s}^{-1}$ & $a_{t}^{-1}$ & $\kappa$ & $m_{\mathrm{J} / \psi}^{T=0}$ \\
\hline $32^{3} \times 64,32,22,16$ & 6.10 & $2.03 \mathrm{GeV}$ & $8.12 \mathrm{GeV}$ & 0.112 & $3.10 \mathrm{GeV}$ \\
\hline \hline
\end{tabular}

Table 1: Parameter set used in this study. We adopt the parameters for clover action in Ref. [9].

masses are taken into account. In Euclidian space-time, the potential of the $J / \psi$ channel at finite temperature is extracted from the following Schrödinger-type equation with the finite quark masses,

$$
\left[-\partial_{\tau}+\frac{\Delta}{2 \mu}-V(\tau, \vec{r} ; T)-2 m\right] G_{E}(\tau, \vec{r})=0
$$

where $\mu$ is the reduced mass and $G_{E}(\tau, \vec{r})$ is given by

$$
G_{E}(\tau, \vec{r}) \equiv \sum_{\vec{x}}\left\langle\bar{q}(\tau, \vec{r}+\vec{x}) \Gamma q(\tau, \vec{x}) \sum_{\vec{y}, \vec{z}} \bar{q}(0, \vec{y}) \Gamma q(0, \vec{z})\right\rangle
$$

with the Coulomb gauge and $\Gamma=\gamma_{\mu}$. Calculating the correlator in Eq. (2.6) on the lattice, $V(\tau, \vec{r} ; T)$ is inversely extracted by Eq. (2.5). With this formalism, we can naively expect that the real part of the real-time potential $V(t, \vec{r} ; T)$ is obtained. Simulations for $1 \ll \tau / a<\beta / 2$ with $\beta \equiv 1 / T$ are shown in the next section.

\section{Simulation setup and numerical results}

In this section, we show the simulation setup. The setup used in this study is same as in Ref. [9] except for lattice sizes. We adopt the anisotropic standard plaquette action for gauge fields,

$$
S_{G}=\frac{\beta}{N_{c}} \frac{1}{\gamma_{G}} \sum_{s, i<j \leq 3} \operatorname{Re} \operatorname{Tr}\left\{1-P_{i j}(s)\right\}+\frac{\beta}{N_{c}} \gamma_{G} \sum_{s, i \leq 3} \operatorname{Re} \operatorname{Tr}\left\{1-P_{i 4}(s)\right\},
$$

with $\beta \equiv 2 N_{c} / g^{2}=6.10$ and $\gamma_{G}=3.2103$, where $P_{\mu \nu}$ is the plaquette operator. The resulting lattice spacing in spatial direction is $a_{s}^{-1}=2.03 \mathrm{GeV}$ and in temporal direction $a_{t}^{-1}=8.12 \mathrm{GeV}$.

As for quarks, we adopt the anisotropic clover action, $S_{F} \equiv \sum_{x, y} \bar{\psi}(x) K_{\text {clover }}(x, y) \psi(y)$, where $K_{\text {clover }}(x, y)$ is defined as

$$
\begin{aligned}
K_{\text {clover }}(x, y) \equiv & \delta_{x, y}-\kappa_{t}\left\{\left(1-\gamma_{4}\right) U_{4}(x) \delta_{x+\hat{4}, y}+\left(1+\gamma_{4}\right) U_{4}^{\dagger}(x-\hat{4}) \delta_{x-\hat{4}, y}\right\} \\
& -\kappa_{s} \sum_{i}\left\{\left(r-\gamma_{i}\right) U_{i}(x) \delta_{x+\hat{i}, y}+\left(r+\gamma_{i}\right) U_{i}^{\dagger}(x-\hat{i}) \delta_{x-\hat{i}, y}\right\} \\
& -\kappa_{s} c_{E} \sum_{i} \sigma_{i 4} F_{i 4} \delta_{x, y}-r \kappa_{s} c_{B} \sum_{i<j} \sigma_{i j} F_{i j} \delta_{x, y} .
\end{aligned}
$$

The improvement of the action is performed by the replacement, $U_{i}(x) \rightarrow U_{i}(x) / u_{s}, U_{4}(x) \rightarrow U_{4}(x) / u_{t}$, where $u_{s}=0.8059$ and $u_{t}=0.9901$ are the mean-field values of the spatial and the temporal link variables in the setup, respectively. The requirement of keeping the Lorentz symmetry up to $O\left(a^{2}\right)$ leads to $r=a_{t} / a_{s}, c_{E}=1 /\left(u_{s} u_{t}^{2}\right), c_{B}=1 / u_{s}^{3}$ and $\gamma_{F} \equiv\left(u_{t} \kappa_{t}\right) /\left(u_{s} \kappa_{s}\right)=a_{s} / a_{t}$ [9]. The hopping parameter in the isotropic lattice, $\kappa=\left(1 /\left(u_{s} \kappa_{s}\right)-2\left(\gamma_{F}+3 r-4\right)\right)^{-1}=0.112$, is adjusted to reproduce the $J / \psi$ mass $m_{J / \psi}=3.1 \mathrm{GeV}$ at zero temperature. The lattice sizes are $32^{3} \times 64,32,22,16$, which 

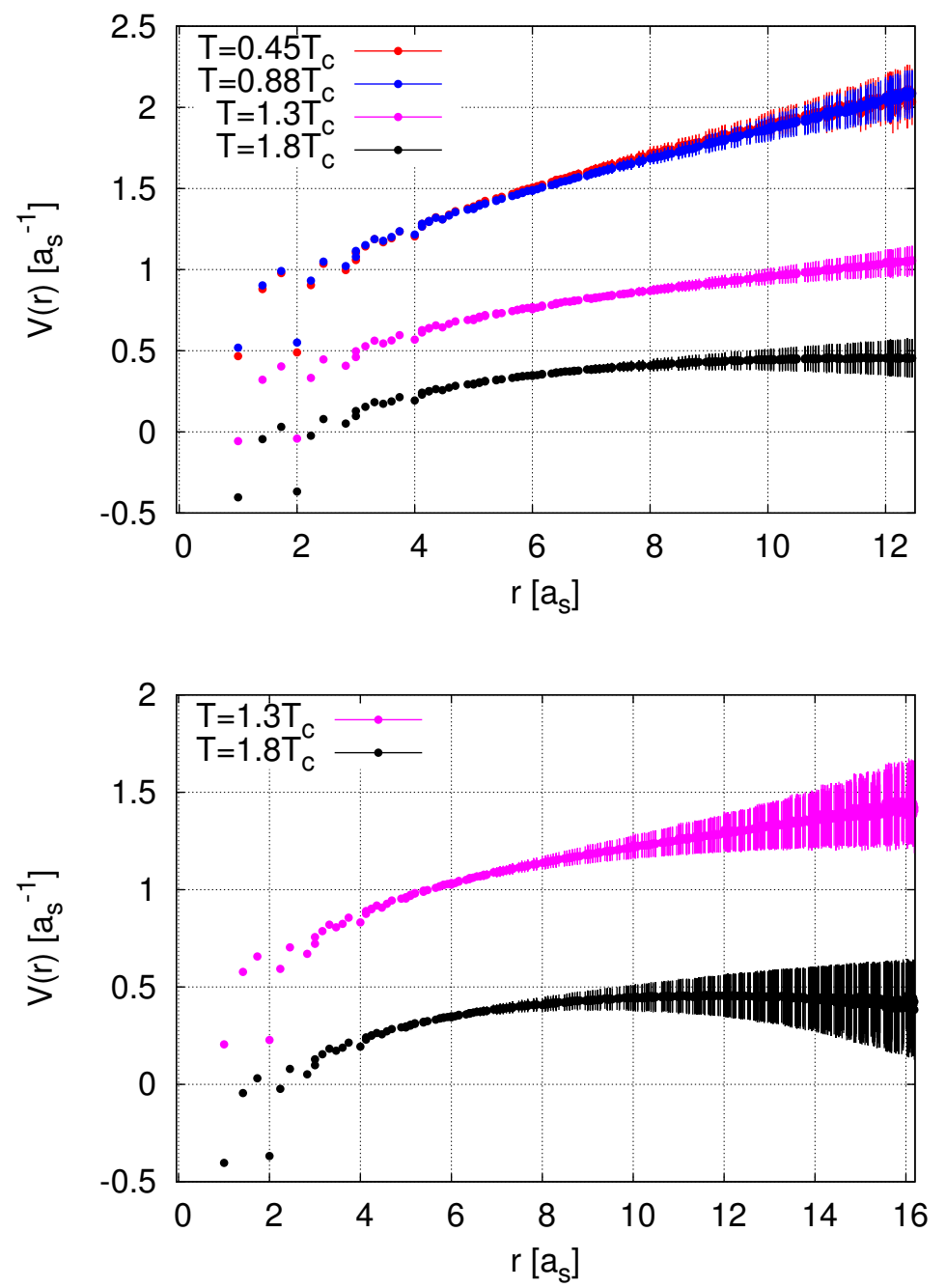

Figure 1: [Upper figure] Temperature dependence of the inter-quark potential (red: $0.45 T_{c}$, blue: $0.88 T_{c}$, magenta: $1.3 T_{c}$, black: $1.8 T_{c}$ ). [Lower figure] Magnified figure of the upper one for the potentials above $T_{c}$.

correspond to $0.45,0.8,1.3,1.8 T_{c}$, respectively. The configuration numbers used in this study are $N_{\text {conf }}=30$ for $32^{3} \times 64$ and $32^{3} \times 32$ lattices and $N_{\text {conf }}=50$ for $32^{3} \times 22$ and $32^{3} \times 16$ lattices. To calculate point separated correlation for quarks, we adopt Coulomb gauge fixing. The parameters are summarized in Table 1.

In the upper figure of Fig. 1, we show the real part of an inter-quark potential derived from Eq. (2.5) and (2.6). Red, blue, magenta and black symbols correspond to the data at $T=0.45,0.88,1.3$ and $1.8 T_{c}$, respectively. Below $T_{c}$, the potential has moderate temperature dependence: they almost coincide and have the linear confinement part with almost the same string tension at zero temperature. On the other hand, above $T_{c}$, the linear part becomes small. The lower figure of Fig. 1 is the magnified figure of the upper one for the potentials above $T_{c}$. At $1.8 T_{c}$, the potential becomes flat at long distance. At $1.3 T_{c}$, the small amount of linear part seems to remain. However, more statistics 
are needed to judge whether there remains linear part or not at $1.3 T_{c}$. The decrease of a linear part of the potential may reflect the deconfinement transition, which is implemented in mesons at high temperatures.

\section{Discussion and summary}

We have studied inter-quark potentials with finite quark masses at finite temperature from NBS amplitudes. We employ the Schrödinger equation in Eq.(2.5) with the correlator in Eq.(2.6), which is an extension of the study by Laine et al. and Beraudo et al. to the case of the finite quark masses. The obtained potentials show moderate temperature dependence below $T_{c}$. On the other hand, above $T_{c}$, the strong temperature dependence is observed. The linear confinement potential vanishes already at $T=1.8 T_{c}$. Although the simulations with more statistics are needed, it is, however, noticed that the potentials are directly obtained from the NBS amplitudes at finite temperature and reflect the changes of the meson properties.

Since, in Ref. [12], they point out that the free energy of $\bar{Q} Q$ in Coulomb gauge, which is the potential in imaginary-time direction at $\tau=\beta$, coincides with the real part of a potential in real-time direction at large $t$ for QED case, as is mentioned in Sec. 2, we expect that the real part of the real-time $\bar{Q} Q$ potential with finite quark masses is extracted. Therefore, we anticipate that the potential in our formalism in heavy quark limit would coincide with that in real-time direction with $t \rightarrow \infty$, although the calculation is the QED case in [12].

On the other hand, the authors of Ref. [12] show that the information of soft collisions (Landau damping) is lost in a potential in imaginary-time direction. To obtain the information, we have to perform an analytic continuation of the correlator in Eq. (2.6) obtained in lattice QCD to real time. Such an analytic continuation of numerical data is actually challenging. A possible way of a calculation of such a real-time correlator in lattice QCD is proposed in Ref. [13], where the authors define a real-time potential at finite temperature in the context of Wilson loop. The obtained potential has not only a real part but also a imaginary part which would originate from the Landau damping. If we perform analytic continuation of Euclidean correlator in Eq. (2.6) to real-time by using spectral representation and MEM same as in Ref. [13], not only the real part but also the imaginary part of the $\bar{Q} Q$ potentials with finite quark masses could be extracted.

\section{Acknowledgments}

We thank S. Aoki, T. Hatsuda, N. Ishii, T. Kawanai, S. Sasaki and the members of HAL QCD Collaboration for useful discussions and comments. The calculations were performed by using NEC-SX9 at Osaka University and partly the RIKEN Integrated Cluster of Clusters (RICC) facility.

\section{References}

[1] Y. Ikeda and H. Iida, Quark-anti-quark potentials from Nambu-Bethe-Salpeter amplitudes on lattice, arXiv:1102.2097; The $\bar{q}-q$ potentials from Nambu-Bethe-Salpeter amplitudes on lattice, PoS

LATTICE2010 (2010) 143. 
[2] N. Ishii, S. Aoki and T. Hatsuda, The Nuclear Force from Lattice QCD, Phys. Rev. Lett.99 (2007) 022001 .

[3] T. Kawanai and S. Sasaki, Interquark potential with finite quark mass from lattice QCD, Phys. Rev. Lett. 107 (2011) 107; Interquark potential for the charmonium system with almost physical quark masses, arXiv:1111.0256.

[4] T. Barnes, S. Godfrey and E.S. Swanson, Higher charmonia, Phys. Rev. D72 (2005) 054026.

[5] T. Umeda, K. Nomura and H. Matsufuru, Charmonium at finite temperature in quenched lattice QCD, Eur. Phys. J.C39S1 (2005) 9.

[6] M. Asakawa and T. Hatsuda, $J / \psi$ and $\eta_{c}$ in the deconfined plasma from lattice QCD, Phys. Rev. Lett.92 (2004) 012001.

[7] S. Datta, F. Karsch, P. Petreczky and I. Wetzorke, Behavior of charmonium systems after deconfinement, Phys. Rev. D69 (2004) 094507.

[8] T. Umeda, A constant contribution in meson correlators at finite temperature, Phys. Rev. D75 (2007) 094502.

[9] H. Iida, T. Doi, N. Ishii, H. Suganuma and M. Oka, Charmonium properties in deconfinement phase in anisotropic lattice QCD, Phys. Rev. D74 (2006) 074502.

[10] P. Gubler, K. Morita and M. Oka, Charmonium spectra at finite temperature from QCD sum rules with the maximum entropy method, Phys. Rev. Lett.107 (2011) 092003.

[11] M. Laine, O. Philipsen, P. Romatschke and M. Tassler, Real-time static potential in hot QCD, JHEP0703 (2007) 054.

[12] A. Beraudo, J.P. Blaizot and C. Ratti, , Real and imaginary-time quarkonium correlators in an hot plasma, PoS CONFINEMENT8 (2008) 117: Real and imaginary-time $Q$ anti- $Q$ correlators in a thermal medium, Nucl. Phys. A806 (2008) 312.

[13] A. Rothkopf, T. Hatsuda and S. Sasaki, Complex Heavy-Quark Potential at Finite Temperature from Lattice $Q C D$, arXiv:1108.1579. 